\title{
Calcul de l'écoulement transitoire laminaire d'un fluide viscoélastique dans une conduite en matériau élastique par une méthode mixte
}

\author{
Abdelouaheb Djemili, Nassereddine Zeghib et Kamel Chaoui ${ }^{\mathrm{a}}$ \\ Laboratoire de Recherche en Mécanique des Matériaux et Maintenance Industrielle (LR3MI), Département de Génie Mécanique, \\ Faculté des Sciences de l'Ingénieur, Université Badji Mokhtar, BP 12, Annaba 23000, Algérie
}

Reçu le 19 mars 2007, accepté le 31 août 2007

\begin{abstract}
Résumé - L'interaction entre un fluide en écoulement et les conduites de transport est en rapport avec le niveau des contraintes appliquées ainsi que la durée de vie souhaitée de la structure. L'approche numérique développée dans cette étude est basée sur une modélisation globale du fluide et de la déformation de la conduite en écoulement transitoire. La contrainte tangentielle de viscosité est exprimée par un développement polynomial mixte représentant la variation du profil radial de la vitesse du fluide. La résolution du système d'équations mis au point a été menée à partir de la méthode des caractéristiques couplée avec les différences finies. Dans le cas d'un fluide newtonien, les résultats obtenus ont pu être comparés avec les données expérimentales de Holmboe et celles théoriques de Zielke. Cette comparaison a permis de confirmer la validité du schéma de calcul utilisé. Une extension des calculs de manière analogue a été réalisée pour la même installation dans le cas d'un fluide viscoélastique linéaire.
\end{abstract}

Mots clés : Écoulement transitoire / interaction fluide structure / fluide viscoélastique / contrainte de cisaillement / méthode des caractéristiques

\begin{abstract}
Laminar transient flow analysis of a viscoelastic fluid in an elastic material duct using a mixed method. The interaction between a flowing fluid and the transmission mains is in relation with the applied pressure level as well as the sought structure lifetime. The numerical approach developed in this study is based on a global modeling of the fluid and the deformation of the duct under transient conditions. The viscous tangential stress is expressed using a mixed polynomial development representing the variation of the radial profile of the fluid speed. The resolution of the obtained system of equations is carried out using the method of the characteristics coupled with the finite differences method. In the case of a Newtonian fluid, the results were compared to the experimental data of Holmboe and the theoretical ones of Zielke. This comparison allowed confirming the validity of the adopted calculation scheme. An extension of calculations in a similar way was carried out for the same installation in the case of a linear viscoelastic fluid.
\end{abstract}

Key words: Transient flow / fluid-structure interaction / viscoelastic fluid / shear stress / characteristics method

\section{Introduction}

De nos jours, il est admis qu'un écoulement en régime transitoire est caractérisé par des surpressions suivies de dépressions dans le temps pouvant conduire inéluctablement à une fatigue des matériaux et éventuellement à une rupture prématurée des conduites. De nombreux travaux ont été consacrés à l'étude des écoulements transitoires de fluides newtoniens en

\footnotetext{
a Auteur pour correspondance : chaoui@univ-annaba.org
}

conduites élastiques et des méthodes de calcul adaptées à ces problèmes ont été mises au point [1-3]. Toutefois, une meilleure représentation de la contrainte de cisaillement pariétale instationnaire est recherchée car les modèles mathématiques restent en deçà du résultat voulu. Dans ce domaine d'interaction fluide-structure, il convient de citer les travaux de Zielke [4], Holmboe et Rouleau [5], Bergant et al. [6] ainsi que l'étude de Prado et Larreteguy [7]. Pour les fluides non-newtoniens, Gally et Rieutord ont traité divers types d'écoulements par une transformation intégrale [8]. Les autres travaux ont été orientés vers 


\section{Nomenclature}

$C_{\mathrm{f}}$ : vitesse de propagation des perturbations (fluide)

$C_{\mathrm{m}}$ : vitesse de propagation d'ondes (matériau)

$E$ : module d'élasticité du matériau, GPa

$e$ : épaisseur, $\mathrm{m}$

$K$ : inverse du coefficient de compressibilité du fluide

$L$ : longueur, $\mathrm{m}$

$N$ : nombre d'éléments

$R$ : rayon, $\mathrm{m}$

$P$ : pression, $\mathrm{Pa}$

$S_{1}$ : temps réduit de relaxation du fluide

$S_{2}$ : temps réduit de retard du fluide

$T_{\mathrm{f}}$ : contrainte de cisaillement pariétale, $\mathrm{Pa}$

$t:$ temps, $\mathrm{s}$

$\stackrel{\circ}{u}$ : vitesse de déplacement axiale, $\mathrm{m} . \mathrm{s}^{-1}$

$V$ : vitesse du fluide, $\mathrm{m} . \mathrm{s}^{-1}$

$x:$ distance, $\mathrm{m}$

$\psi(t)$ : fonction de relaxation

$W(t)$ : fonction poids

$\tau:$ temps réduit

$\sigma$ : contrainte axiale, $\mathrm{Pa}$

$\nu$ : coefficient de Poisson

$\rho:$ masse volumique du fluide, $\mathrm{kg} \cdot \mathrm{m}^{-3}$

$\rho_{\mathrm{m}}$ : masse volumique du matériau, $\mathrm{kg} \cdot \mathrm{m}^{-3}$

$\mu_{\mathrm{f}}$ : viscosité dynamique, $\mathrm{kg} \cdot \mathrm{m}^{-1} \cdot \mathrm{s}^{-1}$

$\nu_{\mathrm{f}}:$ viscosité cinématique, $\mathrm{m}^{2} \cdot \mathrm{s}^{-1}$

$\lambda_{1}:$ temps de relaxation, $\mathrm{s}$

$\lambda_{2}$ : temps de retard, $\mathrm{s}$

$\delta(t)$ : fonction de Dirac

des aspects rhéologiques pour définir les lois de comportement des fluides $[9,10]$. En ce qui concerne les méthodes de résolution numérique des systèmes d'équations qui prennent en compte la vitesse de l'écoulement et la pression couplée à une loi de comportement du matériau, on cite le cas de l'étude de Dorvaux qui a développé une méthode de calcul en se basant sur l'hypothèse de la conduite formée d'un ensemble d'anneaux juxtaposés indépendants en négligeant l'effet de Poisson. Cependant, cette approche offre la possibilité de traiter les écoulements de fluides viscoélastiques linéaires en conduite viscoélastique [11].

L'objet de la présente étude est d'établir un système d'équations régissant l'écoulement instationnaire d'un fluide viscoélastique linéaire dans une conduite en matériau élastique en considérant l'effet de Poisson par un couplage dynamique entre le fluide et la conduite. Le système d'équations différentielles aux dérivées partielles décrivant ce phénomène est résolu par la méthode des caractéristiques couplée à celle des différences finies. En plus, la représentation de la contrainte de cisaillement à la paroi par un développement polynomial mixte doit permettre un gain de temps de calcul et de place mémoire. La combinaison entre cette méthode de calcul et la représentation spécifique de la contrainte pariétale permet de traiter des conditions aux limites très variées.

\section{Formulation mathématique}

\subsection{Système d'équations}

Le système d'équations du type hyperbolique (1) est établi pour un écoulement de fluide dans une conduite en matériau élastique, linéaire, isotrope et dans l'hypothèse des coques minces en petites déformations [1-3]. L'inertie radiale de la paroi est négligée devant les autres termes. Ce système d'équations prend en compte l'ensemble des variables en fonction de la distance $x$ et du temps $t$, incluant le couplage dynamique entre le fluide et la paroi de la conduite :

$$
\left\{\begin{array}{l}
\rho \frac{\partial V}{\partial t}+\frac{\partial P}{\partial x}=\frac{4}{D} T_{\mathrm{f}} \\
\frac{\partial \sigma}{\partial x}-\rho_{\mathrm{m}} \frac{\partial \stackrel{\circ}{u}}{\partial t}=0 \\
\left(\frac{1}{K}+\frac{2 R\left(1-\nu^{2}\right)}{e E}\right) \frac{\partial P}{\partial t}-2 \nu \frac{\partial \stackrel{\circ}{\partial x}}{\partial x}+\frac{\partial V}{\partial x}=0 \\
\frac{\partial \sigma}{\partial t}-E \frac{\partial \stackrel{\circ}{\partial}}{\partial x}-\frac{R \nu}{e} \frac{\partial P}{\partial t}=0
\end{array}\right\}
$$

où $K$ est l'inverse du coefficient de compressibilité du fluide alors que $T_{\mathrm{f}}$ est la contrainte de cisaillement pariétale. Cette dernière dépend d'une fonction poids qui décrit implicitement le caractère bidimensionnel du mouvement local du fluide.

\subsection{Détermination de la contrainte de cisaillement pariétale}

Zielke a établi, dans le cas du fluide newtonien, que la contrainte de cisaillement $T_{\mathrm{f}}$ peut être exprimée à l'aide d'une fonction poids $W(t)$ [4]. Par la suite, Dorvaux a montré qu'il en est de même pour certains fluides viscoélastiques et a donné une application pour le cas d'un fluide d'Oldroyd à trois paramètres [11]. La fonction de relaxation du fluide d'Oldroyd $[9,11]$ est exprimée par la relation suivante :

$$
\psi(t)=\mu_{\mathrm{f}}\left[\frac{\lambda_{2}}{\lambda_{1}} \delta(t)+\left(1-\frac{\lambda_{2}}{\lambda_{1}}\right) \frac{e^{-t / \lambda_{1}}}{\lambda_{1}}\right]
$$

où $\mu_{\mathrm{f}}$ et $\nu_{\mathrm{f}}$ représentent respectivement les viscosités dynamique et cinématique du fluide, $\lambda_{1}$ est le temps de relaxation alors que $\lambda_{2}$ est le temps de retard avec $\left(\lambda_{1}>\lambda_{2}\right)$ et $\delta(t)$ la fonction de Dirac. Deux cas découlent de l'équation (2) à savoir $\lambda_{1}=\lambda_{2}$ et $\lambda_{2}=0$ qui caractérisent respectivement le fluide newtonien et le fluide de Maxwell. La connaissance de la fonction de relaxation $\psi(t)$ du fluide permet d'évaluer la contrainte de cisaillement pariétale avec l'équation :

$T_{\mathrm{f}}(x, t)=-\frac{8 \rho \nu_{\mathrm{f}}}{D}\left(V(x, t)+\frac{1}{2} \int_{0}^{t} W\left(t-t^{\prime}\right) \frac{\partial V}{\partial t^{\prime}} \mathrm{d} t^{\prime}\right)$

où le premier terme représente les effets associés au régime permanent. Le second terme est le produit de convolution qui correspond aux phénomènes instationnaires et fait intervenir la loi de comportement du fluide. 


\section{Méthode de résolution}

\subsection{Résolution mathématique}

Le système d'équations (1) aux dérivées partielles se prête bien à une résolution par la méthode des caractéristiques. Ce système admet quatre directions caractéristiques [3] :

$$
\begin{aligned}
& \beta_{1,2}= \pm C_{\mathrm{f}}= \pm \frac{a_{\mathrm{f}}}{\bar{J}} Q_{1} \\
& \beta_{3,4}= \pm C_{\mathrm{m}}= \pm \frac{a_{\mathrm{m}}}{\bar{J}} Q_{2}
\end{aligned}
$$

où $C_{\mathrm{f}}$ représente la vitesse de propagation des perturbations de pression et de vitesse dans le milieu fluide alors que $C_{\mathrm{m}}$ est la vitesse de propagation de l'onde des contraintes et déformations longitudinales dans le matériau de la conduite (milieu solide). Les différents coefficients et paramètres sont calculés avec les équations :

$$
\begin{gathered}
a_{\mathrm{f}}=\left(\frac{K}{\rho}\right)^{\frac{1}{2}} \\
a_{\mathrm{m}}=\left(\frac{K}{\rho_{\mathrm{m}}}\right)^{\frac{1}{2}} \\
\tilde{J}=\left(1+\frac{2 R K}{e E}\left(1-\nu^{2}\right)\right)^{\frac{1}{2}} \\
Q_{1,2}=\left(\left(\frac{1+q}{2}\right) \pm \sqrt{\frac{(1-q)^{2}}{2}+\frac{2 R \nu^{2} \rho}{e \rho_{\mathrm{m}}}}\right)^{\frac{1}{2}} \\
q=\left(1+\frac{2 R K}{e E}\right) \frac{a_{\mathrm{m}}^{2}}{a_{\mathrm{f}}^{2}}
\end{gathered}
$$

Les relations de compatibilité sont exprimées par :

- le long de $\frac{\mathrm{d} x}{\mathrm{~d} t}= \pm C_{\mathrm{f}}$ (caractéristiques $C_{\mathrm{f}}^{+}$et $C_{\mathrm{f}}^{-}$) :

$\frac{\mathrm{d} P}{\mathrm{~d} t} \pm \rho_{\mathrm{f}} \frac{\mathrm{d} V}{\mathrm{~d} t} \pm \rho_{\mathrm{m}} C_{\mathrm{f}} G_{\mathrm{f}} \frac{\mathrm{d} \dot{u}}{\mathrm{~d} t}-G_{\mathrm{f}} \frac{\mathrm{d} \sigma}{\mathrm{d} t}= \pm C_{\mathrm{f}} \frac{4 T_{\mathrm{f}}}{D}$

- le long de $\frac{\mathrm{d} x}{\mathrm{~d} t}= \pm C_{\mathrm{m}}$ (caractéristiques $C_{\mathrm{m}}^{+}$et $\left.C_{\mathrm{m}}^{-}\right)$:

$\frac{\mathrm{d} P}{\mathrm{~d} t} \pm \rho C_{\mathrm{m}} \frac{\mathrm{d} V}{\mathrm{~d} t} \pm \rho_{\mathrm{m}} C_{\mathrm{m}} G_{\mathrm{m}} \frac{\mathrm{d} \dot{u}}{\mathrm{~d} t}-G_{\mathrm{m}} \frac{\mathrm{d} \sigma}{\mathrm{d} t}= \pm C_{\mathrm{m}} \frac{4 T_{\mathrm{f}}}{D}$

avec :

$$
G_{\mathrm{f}}=\frac{2 \nu \rho C_{\mathrm{f}}^{2}}{\rho_{\mathrm{m}}\left(a_{\mathrm{m}}^{2}-C_{\mathrm{f}}^{2}\right)}
$$

et

$$
G_{\mathrm{m}}=\frac{2 \nu \rho C_{\mathrm{m}}^{2}}{a_{\mathrm{m}}\left(a_{\mathrm{m}}^{2}-C_{\mathrm{m}}^{2}\right)}
$$

À ce système d'équations qui permet de calculer les évolutions des variables $P, V \stackrel{\circ}{u}$ et $\sigma$ en tout point de la conduite, il faut adjoindre les conditions aux limites et initiales.

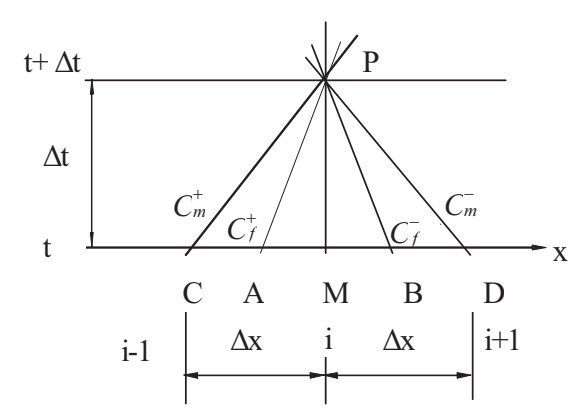

Fig. 1. Discrétisation et droites caractéristiques dans le plan $(x, t)$.

\subsection{Méthode de résolution numérique}

Parmi les méthodes de résolution numérique du système d'équations (1), la méthode de Hartree qui considère un maillage régulier a été retenue [12]. Cette méthode est combinée avec la méthode des différences finies. Un cas spécifique a été adopté de façon à éviter toute interpolation pour la caractéristique $C_{\mathrm{m}}$ concernant le matériau (Fig. 1). Le pas de temps doit satisfaire le critère de stabilité de Courant-Friedrich [12] :

$$
\Delta t \leqslant \frac{\Delta x}{\left| \pm C_{\mathrm{m}}\right|}
$$

\subsubsection{Calcul de $P, V, \stackrel{\circ}{u}$, et $\sigma$}

La résolution des équations de compatibilité le long des courbes $C_{\mathrm{f}}^{ \pm}$et $C_{\mathrm{m}}^{ \pm}$écrites en terme de différences finies permet de calculer, pour un point courant, la pression, la vitesse du fluide, la vitesse de déplacement et la contrainte dans la conduite [13].

\subsubsection{Conditions aux limites et initiales}

Ces conditions portent, selon le cas considéré, sur la valeur imposée de la vitesse ou de la pression et/ou de la vitesse de déplacement. Les valeurs initiales de ces paramètres sont celles du régime permanent juste avant l'application de la perturbation.

\subsubsection{Calcul du terme de frottement $\left(T_{\mathrm{f}}\right)$}

Pour les fluides newtoniens, bien qu'une bonne représentation de la fonction poids $W(t)$ ait été donnée par Zielke, le calcul du produit de convolution au pas de temps considéré nécessite la connaissance des vitesses à tous les pas de temps précédents en chaque nœud. L'espace mémoire nécessaire au calcul devient alors prohibitif. Pour contourner cette difficulté, Trikha [14], Suzuki [15] et Schohl [16] ont proposé de représenter $W(t)$ à l'aide d'une série exponentielle. Quand $t$ tend vers zéro, la représentation par une série limitée d'exponentielles n'est 
pas satisfaisante et conduit à une sous-évaluation de la contribution de la vitesse instantanée dans le calcul du terme de frottement. Cette erreur est cumulative et peut devenir sensible pour les temps assez grands. Afin de minimiser cette erreur, cette fonction va être exprimée par une représentation polynomiale mixte :

$$
\begin{gathered}
W(\tau)=\left\{\sum_{j=1}^{p} C_{j} \tau^{(j-2) / 2}\right\} \quad \text { pour } 0<\tau \leqslant \Delta \tau \\
W(\tau)=\left\{\sum_{i=1}^{n} b_{i} \cdot I_{i}^{k}\right\} \quad \text { pour } \tau>\Delta \tau
\end{gathered}
$$$$
T_{\mathrm{f}}^{k+1}=-\frac{8 \rho \nu_{\mathrm{f}}}{D}\left\{V^{k+1}+\frac{1}{2} C\left(V^{k+1}-V^{k}\right)+\frac{1}{2} \sum_{i=1}^{n} b_{i} I_{i}^{k}\right\}
$$

avec le temps réduit $\tau$ exprimé par $\tau=t \frac{\nu_{\mathrm{f}}}{R^{2}}$ et le coefficient $C$ exprimé par :

$$
C=\left\{\sum_{j=1}^{p} C_{j} \cdot \tau^{(j-2) / 2}\right\}
$$

Les coefficients $C_{j}$ sont donnés par les relations suivantes [11] :

$$
\begin{array}{cc}
C_{1}=\frac{1}{2 \sqrt{\pi}}\left(\frac{S_{2}}{S_{1}}\right)^{1 / 2} & (11 \mathrm{~b}) \\
C_{2}=\frac{3}{4} \frac{S_{2}}{S_{1}}-2 & (11 \mathrm{c}) \\
C_{3}=\frac{1}{2 \sqrt{\pi}}\left(\frac{S_{2}}{S_{1}}\right)^{1 / 2}\left\{\frac{15}{4} \frac{S_{2}}{S_{1}}+\frac{1}{2}\left(\frac{1}{S_{2}}-\frac{1}{S_{1}}\right)\right\} & (11 \mathrm{~d}) \\
C_{5}=\frac{1}{192 \sqrt{\pi}} \frac{S_{2}}{S_{1}}\left\{15 \frac{S_{2}}{S_{1}}+12\left(\frac{1}{S_{2}}-\frac{1}{S_{1}}\right)\right\} & (11 \mathrm{e}) \\
+360\left(\frac{S_{1}-S_{2}}{S_{1}}\right\}^{1 / 2}\left\{135\left(\frac{S_{2}}{S_{1}}\right)^{2}+\frac{48}{S_{1}^{2}}-\frac{32}{S_{1} S_{2}}-\frac{16}{S_{2}^{2}}\right. \\
+C_{6}=\frac{1}{128} \frac{S_{2}}{S_{1}}\left\{48\left(\frac{1}{S_{1}^{2}}-\frac{1}{S_{1}} S_{2}\right)\right. \\
\left.+120\left(\frac{1}{S_{2}}-\frac{S_{2}}{S_{1}^{2}}\right)-45\left(\frac{S_{2}}{S_{1}}\right)^{2}\right\} & (11 \mathrm{~g})
\end{array}
$$

où $S_{1}$ et $S_{2}$ sont les temps réduits de relaxation et de retard du fluide exprimés par les relations :

$$
S_{1}=\lambda_{1} \frac{\nu_{\mathrm{f}}}{R^{2}}
$$

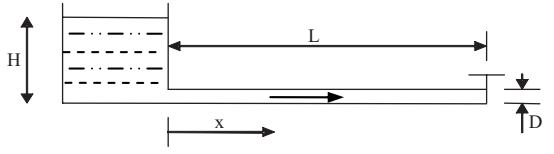

Fig. 2. Schéma de l'installation composée d'un réservoir, d'un tronçon de tube et d'une vanne.

$$
S_{2}=\lambda_{2} \frac{\nu_{\mathrm{f}}}{R^{2}}
$$

Les intégrales $I_{i}^{k}$ suivent la relation de récurrence :

$$
I_{i}^{k+1}=e^{a_{i} \Delta \tau} I_{i}^{k}+\frac{e^{a_{i} \Delta \tau}-1}{a_{i} \Delta \tau}\left(V^{k+1}-V^{k}\right)
$$

avec $I_{i}^{1}=0$.

\section{Applications et résultats}

\subsection{Données viscoélatiques des fluides}

La littérature fournit les coefficients nécessaires pour évaluer le terme de frottement. Pour un fluide newtonien et $S_{1}=S_{2}=0,4$, les coefficients obtenus sont :

$\left\{a_{i}, i=1, \ldots, 7\right\}=\{-26,539 ;-99,026 ;-59,165 ;-2044,344$; $-9592,554 ;-42109,831 ;-171767,684\}$,

$\left\{b_{i}, i=1, \ldots, 7\right\}=\{1,058 ; 2,223 ; 5,184 ; 10,926 ; 23,699$; $47,272 ; 90,975\}$

$\left\{C_{j}, j=1, \ldots, 6\right\}=\{0,282 ;-1,250 ; 1,057 ; 0,937 ; 0,396$; $-0,351\}$.

Dans le cas d'un fluide viscoélastique avec $S_{1}=0,4$ et $S_{2}=0,04$, les coefficients associés sont :

$\left\{a_{i}, i=1, \ldots, 8\right\}=\{-2,5 ;-7,574 ;-128,925 ;-755,222$; $-3303,71215 ;-13821,038$; -58 589,217; - 222405,012$\}$,

$\left\{\mathrm{b}_{i}, i=1, \ldots, 8\right\}=\{-1,800 ; 0,741 ; 0,954 ; 2,180 ; 4,091$; $8,678 ; 16,830 ; 31,154\}$

$\left\{\mathrm{C}_{j}, j=1, \ldots, 6\right\}=\{0,089 ;-1,925 ; 1,037 ; 1,696 ;-10,118$; $21,058\}$.

\subsection{Installation hydraulique}

Afin de valider le modèle proposé, des conditions identiques à celles utilisées par Zielke et Holmboe sont considérées comme dans la figure 2. La conduite est en cuivre avec un coefficient de Poisson de 0,34, une longueur de $36 \mathrm{~m}$ et un diamètre de $0,026 \mathrm{~m}$. La vitesse du fluide est de $0,12 \mathrm{~m} . \mathrm{s}^{-1}$ pour une viscosité cinématique de $39,67 \times 10^{-6} \mathrm{~m}^{2} \cdot \mathrm{s}^{-1}$. Ces conditions imposent un écoulement laminaire.

\subsection{Conditions initiales et aux limites}

Les conditions initiales sont données par la ligne de charge du régime permanent initial. L'écoulement transitoire est réalisé par la fermeture brusque d'une vanne en aval. La pression au niveau du réservoir en amont est 
supposée constante et la vanne est immobile. La conduite est supposée fixée au niveau des points de discrétisation (nœuds du maillage) ce qui se traduit par une vitesse de déplacement axiale nulle.

\subsection{Résultats}

Pour étudier le régime transitoire, la conduite de longueur $L$ est subdivisée en $N$ éléments. La longueur de chaque élément est $\Delta x=L / N$. Un nombre $N=360$, un pas de temps $\Delta t=2,7257 \times 10^{-5} \mathrm{~s}$ ont été choisis afin d'assurer une précision suffisante dans les calculs et un pas de temps en adéquation avec les temps caractéristiques du fluide. Le test de validité numérique réalisé pour $N=720$ a montré que les résultats numériques restent pratiquement inchangés.

\subsubsection{Comportement de la fonction poids $W(\tau)$}

La figure 3 représente les courbes de la fonction poids $W(\tau)$ pour $S_{1}=0,4$ et $S_{2}$ variant de 0 à 0,4 . Pour le cas du fluide newtonien, $S_{1}=S_{2}=0,4$, elle tend vers zéro de façon monotone décroisante quand $\tau$ croît. La contrainte pariétale, qui est en partie représentée par le produit de convolution du terme $\rho \frac{\partial V}{\partial t}$ et de la fonction poids $W(\tau)$, présente donc, pour le fluide newtonien, des effets de mémoire à caractère évanescent associés au terme d'inertie. Ces effets représentent l'influence sur le terme de frottement des variations du profil des vitesses qui interviennent dans les écoulements instationnaires. La décroissance de $W(\tau)$ dans le cas des fluides viscoélastiques est plus rapide que celle du fluide newtonien. Les valeurs des produits de convolution correspondants et, par suite, la contrainte pariétale, sont donc plus faibles que dans le cas du fluide newtonien, ce qui traduit une diminution des effets visqueux comme indiqué dans la figure 4. L'expression de la viscosité instantanée $\left(\nu_{\mathrm{f}} \frac{S_{2}}{S_{1}}\right)$ montre que les fluides vicoélastiques sont moins dissipatifs que le fluide newtonien à cause du rapport $S_{2} / S_{1}$ qui est inférieur à 1 . Cette propriété est en relation avec le caractère élastique qui devient prépondérant quand $S_{2}$ tend vers zéro. La fonction n'est pas strictement monotone décroissante mais elle prend, pour les temps intermédiaires, des valeurs négatives. Ce changement de signe, qui conduit à une inversion de l'orientation de la contrainte pariétale, traduit l'influence des propriétés élastiques de ces fluides sur l'écoulement. Les effets de mémoire, associés au terme d'inertie $\rho \frac{\partial V}{\partial t}$ n'ont un caractère évanescent que globalement c'est-à-dire qu'à des valeurs de $\tau$ importantes, toutes les courbes de $W(\tau)$ tendent vers zéro.

\subsubsection{Cas d'un fluide newtonien $S_{1}=S_{2}=0,4$ )}

La figure 5 représente l'évolution de la hauteur de pression au niveau de l'extrémité aval $(x=L)$. Au début, une augmentation de la pression est enregistrée pendant

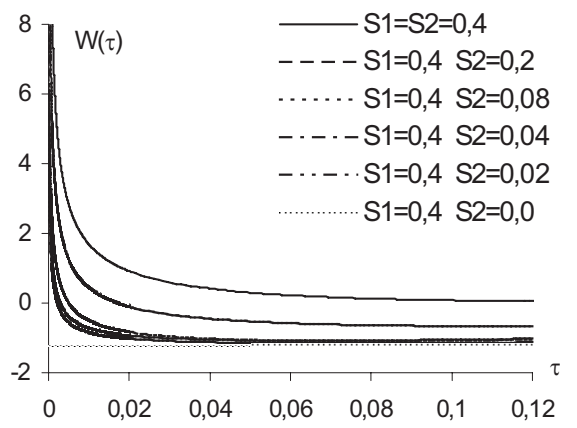

Fig. 3. Évolution de la fonction poids $W(\tau)$ pour différentes valeurs de $S_{1}$ et $S_{2}$.

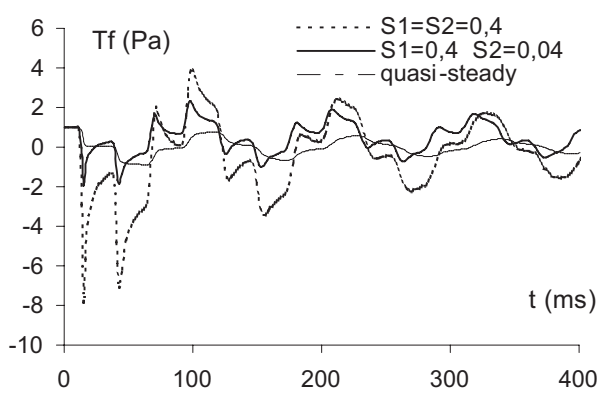

Fig. 4. Contrainte de cisaillement pariétale au milieu de la conduite, i.e. $x=L / 2$.

un temps correspondant à celui de la fermeture de la vanne, puis le premier palier de pression d'une durée d'un temps d'aller-retour d'onde (54,4 ms) apparaît pour une vitesse acoustique dans le fluide de $1324,3 \mathrm{~m} . \mathrm{s}^{-1}$. Il est conclu que le modèle du régime permanent est en deçà du niveau de pression réelle. Pendant toute l'évolution de la pression, il y a un net amortissement des différentes courbes par rapport à celles calculées avec un terme de frottement du régime permanent. Les résultats de calculs obtenus dans cette étude sont comparés avec ceux de la littérature $[4,5]$. Une bonne corrélation est observée entre les différents signaux. Cette concordance entre les résultats confirme la validité de la formulation mathématique proposée et du schéma de calcul utilisé. L'avantage de ce dernier par rapport à celui de Zielke est que le calcul du terme de frottement ne nécessite plus la connaissance de toutes les valeurs antérieures des vitesses ce qui constitue un gain de place mémoire et de temps de calcul.

\subsubsection{Cas de fluide non-newtonien}

Les figures 6 et 7 donnent les résultats de calcul de la hauteur de pression et de la contrainte axiale dynamique à l'extrémité aval de la conduite $(x=L)$ pour les 2 cas étudiés : (a) fluide non-newtonien $\left(S_{1}=0,4, S_{2}=0,04\right)$ et (b) fluide newtonien $\left(S_{1}=S_{2}=0,4\right)$. Ces courbes présentent les aspects typiques du phénomène de coup de bélier mais le caractère viscoélastique du fluide nonnewtonien se traduit par un amortissement plus faible des perturbations de pression et de vitesse. Les résultats 


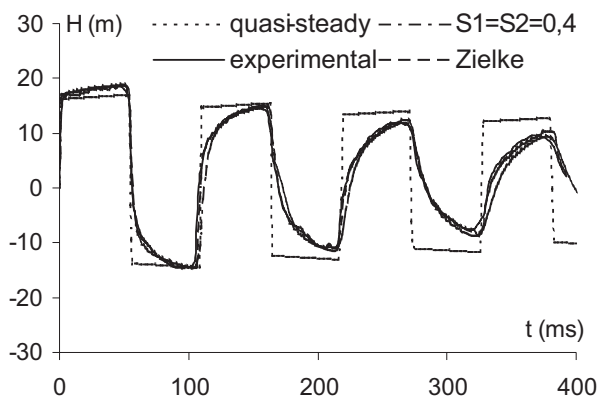

Fig. 5. Hauteur de pression pour le fluide newtonien au niveau de la vanne $(x=L)$.

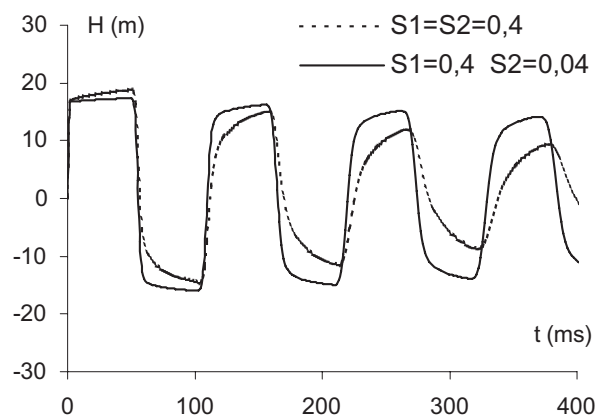

Fig. 6. Hauteur de pression pour le fluide non-newtonien au niveau de la vanne $(x=L)$.

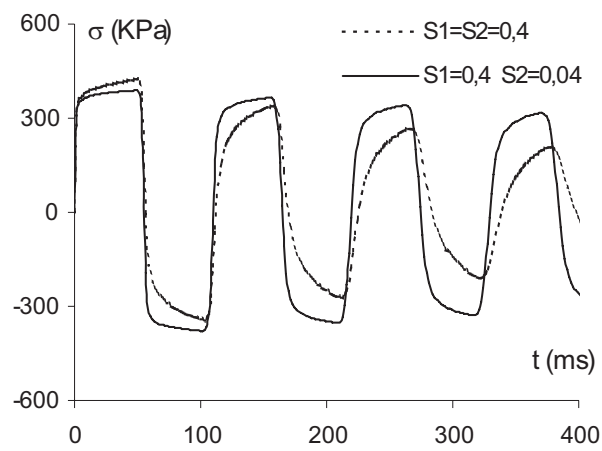

Fig. 7. Variations de contrainte axiale pour le fluide nonnewtonien en $x=L$.

de calcul de la hauteur de pression, de la vitesse du fluide et la contrainte axiale dynamique au milieu de la conduite $(x=L / 2)$ pour les mêmes fluides sont indiqués dans les figures 8-10. Globalement, il est noté les mêmes aspects que dans les figures 6 et 7 . Pour les temps courts, il est vraisemblable que l'écoulement obtenu dans le cas du fluide viscoélastique est assez proche de celui d'un fluide newtonien dont la viscosité serait égale à la viscosité instantanée du fluide viscoélastique étudié.

Cette méthode de calcul ouvre la voie pour l'étude des écoulements de fluides dans des conduites en matériaux polymères. Elle permet aussi de prendre en compte les cas de conditions aux limites très variées comportant des singularités géométriques et directionnelles.

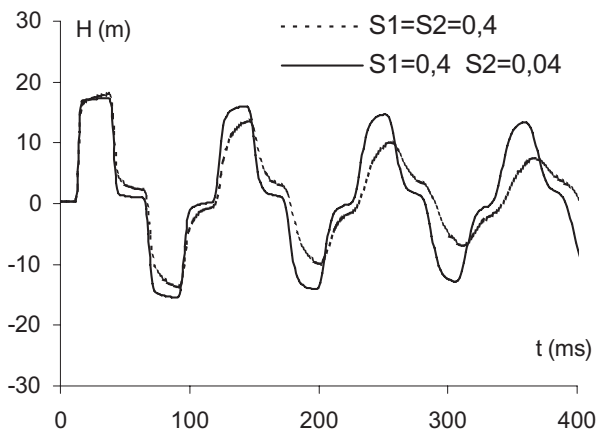

Fig. 8. Hauteur de pression pour le fluide non-newtonien au milieu de la conduite $(x=L / 2)$.

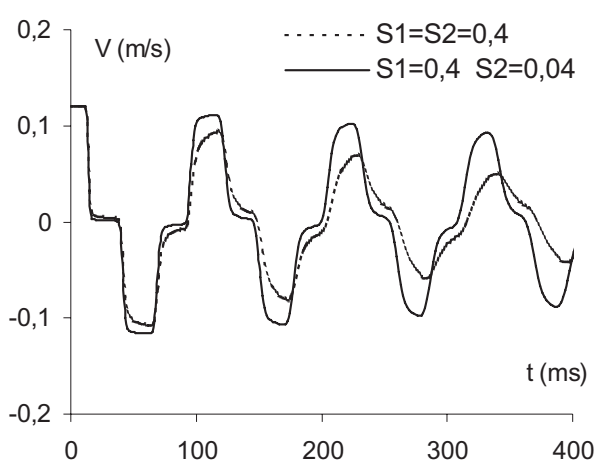

Fig. 9. Vitesse pour le fluide non-newtonien en $x=L / 2$.

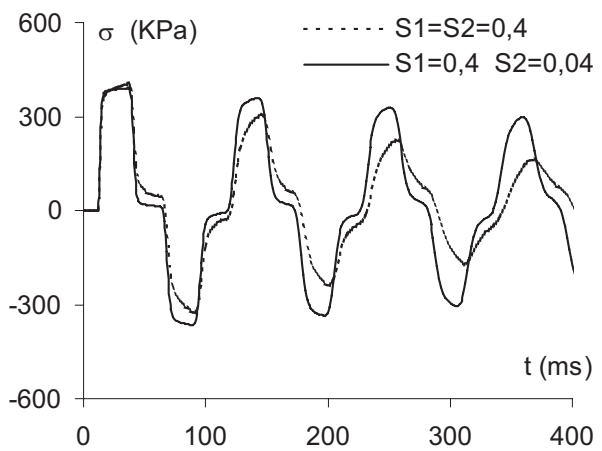

Fig. 10. Contrainte axiale pour le fluide non-newtonien en $x=L / 2$.

\section{Conclusion}

La formulation mathématique proposée a permis d'étudier le phénomène d'écoulement transitoire d'un fluide viscoélastique linéaire dans une conduite en matériau élastique. La contrainte de cisaillement instationnaire a été modélisée par un polynôme mixte. La bonne concordance des résultats numériques avec ceux de la littérature qu'ils soient expérimentaux ou théoriques, confirme l'intérêt pratique de cette modélisation et le schéma de calcul adopté. L'avantage réside dans un gain important de place mémoire et de temps de traitement numérique.

Remerciements. Une partie de ce travail a été réalisée dans le cadre du projet CNEPRU intitulé « Contribution à l'étude 
des écoulements de fluides non-newtoniens dans des conduites en matériaux élastiques ou viscoélastiques » et agréé par le Ministère de l'Enseignement Supérieur et de la Recherche Scientifique sous le code J2301/03/04/00 (LR3MI, Université d'Annaba, Algérie). La validation expérimentale est en cours de réalisation sous la conduite de Pr. N.E. Zeghib de l'équipe Vieillissement et Endommagement des Matériaux.

\section{Références}

[1] A.S. Tijsseling, Fluid-structure interaction in liquid-filled pipe systems : A Review, J. Fluids Struct. 10 (1996) 109146

[2] D.C. Wiggert, Fluid transients in flexible piping systems : a perspective on recent developments, Proceedings of the 18th Symposium on Hydraulic Machinery and Cavitation, Valence, Espagne, 1996, pp. 58-67

[3] A. Djemili, Influence de l'ancrage de la conduite - paroi élastique ou viscoélastique - sur les écoulements en régime transitoire, Premier Séminaire de Mécanique, SMSM, Tome 2, Rabat, Maroc, avril 1993, pp. 275-283

[4] W. Zielke, Frequency-dependent friction in transient pipe flow, J. Basic Eng. ASME 90 (1968) 109-115

[5] E.L. Holmboe, W.T. Rouleau, The Effects of Viscous Shear on Transients in Liquid Lines, J. Basic Eng. ASME 80 (1967) 174-180

[6] A. Bergant, A.R. Simpson, J. Vitkovsky, Developments in unsteady pipe flow friction modeling, J. Hydraulic Res. 39 (2001) 249-256

[7] R. A. Prado, A.E. Larreteguy, A transient shear stress model for the analysis of laminar water-hammer problems, J. Hydraulic Res. 40 (2002) 45-53
[8] M. Gally, E. Rieutord, Influence relative de la viscoélasticité du fluide et de celle de la paroi sur l'évolution des perturbations en conduites, Congrès Français de Mécanique, Marseille, France, septembre 1981

[9] J.G. Oldroyd, On the formulation of rheological equations of state, Proceedings of the Royal Society 200 (1950) 523541

[10] K. Walters, Non-Newtonian effects in some elasticoviscous liquids whose behavior at small rates of shear is characterized by a general linear equation of state, The Quarterly J. Mech. Appl. Math. 15 (1962) 63-76

[11] J.M. Dorvaux, Étude d'une méthode de calcul des écoulements transitoires de fluides viscoélastiques en conduites viscoélastiques, Thèse de Doctorat de $3^{\mathrm{e}}$ cycle, INSA de Lyon, France, 1983

[12] E.B. Wylie, V.L. Streeter, Fluid Transients, FEB Press, Michigan, USA, 1990

[13] A. Djemili, K. Chaoui, Contribution à l'étude des écoulements instationnaires de fluides non-newtoniens dans des conduites en matériau élastique, JEM'03, Annaba, Algérie, 16-17 décembre 2003

[14] A.K. Trikha, An Efficient Method for Simulating Frequency-Dependent Friction in Transient Liquid Flow, J. Fluids Eng. ASME 97 (1975) 97-105

[15] K. Suzuki, T. Taketomi, S. Sato, Improving Zielke's method of simulating frequency-dependent friction in laminar liquid pipe flow, J. Fluids Eng. ASME 113 (1991) 569-573

[16] G.A. Schohl, Improved approximate method for simulating frequency-dependent friction in transient laminar flow, J. Fluids Eng. ASME 115 (1993) 420-424 